cataclysm, comprising a few major basin-forming events, which ended $3.9 \mathrm{Gyr}$ ago? Alternatively, can they be accounted for by a steadily decreasing cratering rate, resulting in a statistically-determined distribution in which individual ages reflect the latest event to reset the Ar clock? Cadogan and Turner favour the calaclysmic theory. Samples from Luna 24 should arrive at Houston in the spring and radiometric results from them are eagerly awaited.

\section{Morphogenesis of the mammary gland}

\section{from John K. Heath}

UNDERSTANDING the mechanisms underlying the formation of organs poses a formidable problem to the developmental biologist. It is known that in most systems interactions between the epithelium of the organ rudiment and its adjacent mesenchyme are involved, but these interactions have proved to be complex. Two recent pieces of work which use the mouse mammary gland, present new experimental approaches which may provide an insight into this problem.

If the mammary epithelium is cultured in contact with mammary mesenchyme the tissues undergo typical mammary morphogenesis and differentiation, but if the epithelium is cultured alone its development is curtailed. If salivary gland is used as the source of mesenchyme, however, the subsequent morphogenesis of the mammary epithelium is typical of the salivary epithelium (Kratochwil Devl Biol. 20, $46 ; 1969$ ), but the biochemical properties of the mammary epithelium in this case, however, are similar to normal mammary glands in situ. Sakakura et al. (Science 194, 1439; 1976) have now taken submandibular salivary gland mesenchyme from mouse embryos 14 days after conception and combined it with mammary epithelium from 16 day post-conception female embryos. After a brief period of culture the combined tissues were transferred to a site under the kidney capsule of adult female mice. Subsequent histological examination of the transplants revealed the typical salivary pattern of morphogenesis. But if the mice carrying the grafts were mated and allowed to give birth and lactate, the ducts in the graft became distended with milk. Biochemical studies showed the transplanted tissues to be producing amounts of the $B$ protein of lactose synthetase similar to those found in lactating mammary glands in situ. These findings show that although the mesenchyme plays an important part in determining the overall form, the cytodifferentiation of the gland is either already determined by 16 days or is independent of the underlying mesenchyme.

The use of hoterologous mesenchyme in recombination experiments is common. But by the time experimental recombinations can be made many interactions involved in the formation of the organ may have occurred. Another problem is that although in teractions can be demonstrated it is hard to tell which tissue takes the lead in determining the subsequent path of development. These difficulties have been circumvented by the exploitation of a mutant gene affecting the differentiation of the mammary gland (Kratochwil and Schwartz Proc. natn. Acad. Sci. U.S.A. 73, 4041; 1976).

The mammary gland of the male can be distinguished from that of the female by the fourteenth day of gestation since it undergoes regression and either disappears completely or remains in a rudimentary state (depending on the strain of mouse used). The mesenchyme surrounds an epitheliat bud and begins to condense around it. The bud then starts to undergo necrosis and the 'stalk' of the bud is cut off. This pattern of regression can be inhibited by destruction of the foetal testes and can be induced in femalcs by administering testosterone. The involvement of exogenous substances such as hormones would seem only to complicate an already difficult situation. However Kratochwil and Schwartz have shown how the action of testosterone can be used as a starting point for unravelling the interactions involved in the process of regression.

They asked whether the hormone exerts its effect on the mesenchyme or the epithelium or both. The key to the solution of this problem was the use of the X-linked mutant 'Testicular Feminisation' ( $\mathrm{Tfm})$, tissues of which are insensitive to testosterone (probably due to a malfunctioning hormone receptor molecule). Although a $\mathrm{X}^{\mathrm{Ttm}} / \mathrm{Y}$ mouse is genetically male it lacks male secondary sex characteristics, and in particular the mammary glands do not show the typical testosterone-induced regnession. Kratochwil and Schwartz combined epithelium and mesenchyme from $X^{\mathrm{Ttm}} / \mathrm{Y}$ and $\mathrm{X} / \mathrm{Y}$ embryos and observed the effects of testosterone on their development. The regression response occurred only if the mesenchyme was wild type. This implies that testosterone initially acts on the mesenchyme and that the subsequent fate of the epithelium is determined by the mesenchyme. An interesting corollary of this observation as the implication that the necrosis of the epithelium is the result of killing by the mesenchyme as apposed to the cell-autonomous suicide typical of cell death in other systems.

The exploitation of appropriate mutants has proved of enormous value in other developmental problems and Kratochwill's work has opened up the possibility of their use in the study of organogenesis. In addition these experiments may lead to the use of ather hormone-triggered tissue interactions for looking at epithelial/mesenchymal interactions.

\section{Hot white dwarfs and the EUV}

\section{from $M$. G. Edmunds}

Apart from the Sun, very few astronomical sources have yet been detected in the extreme ultraviolet (EUV) wavelength region $100-1,000 \AA$. The flight of the Apollo-Soyuz mission in July 1975 provided a platform for a grazing-incidence mirror telescope with a filter photometer, and the detection of two sources outside the Solar System has been reported. Both sources have good positional identification with white dwarf stars, and the second of these (Margon et al. A strophys. J. Lett. 210, 79; 1976) is Feige 24, a well-known spectrophotometric standard at visible wavelengths.

The EUV continuum observations have implied a considerably higher atmospheric temperature for these white dwarfs than had previously been assumed. At optical wavelengths the continuum radiation energy distribution is fairly insensitive to the atmosphere parameters, but model atmosphere calculations suggest that the fluxes in the EUV are more sensitive, especially shortward of the once-ionised helium absorption edge at $229 \AA$, provided that there is a significant helium abundance in the star's atmospheres. The temperature of the other white dwarf HZ43 has been inferred to be as high as $10^{5} \mathrm{~K}$, and it is detected as a very soft X-ray source. Although high for a white dwarf, such a temperature is comparable with temperatures derived for the central stars of planetary nebulae by the Zanstra method-in which the ultraviolet flux from the star is indirectly estimated by observation of optical re-emission of the energy from the ultraviolet radiation absorbed by the nebular gas surrounding the star. Thus it is reasonable that HZ43 is a fairly young white dwarf, still very hot after passing through the planetary nebula stage in which it shed its outer layers during its evolution from a giant.

The EUV observations and ultra- 